CALT-68-2345

CITUSC/01-030

PUPT-2005

hep-th/0108239

\title{
Some interesting violations of the Breitenlohner-Freedman bound
}

\author{
Steven S. Gubser ${ }^{1,2}$ and Indrajit Mitra ${ }^{2}$ \\ ${ }^{1}$ Lauritsen Laboratory of Physics, 452-48 Caltech, Pasadena, CA 91125 \\ ${ }^{2}$ Joseph Henry Laboratories, Princeton University, Princeton, NJ 08544
}

\begin{abstract}
We demonstrate that $A d S_{5} \times T^{p q}$ is unstable, in the sense of Breitenlohner and Freedman, for unequal $p$ and $q$. This settles, negatively, the long-standing question of whether the $T^{p q}$ manifolds for unequal $p$ and $q$ might correspond to non-supersymmetric fixed points of the renormalization group. We also show that the $A d S_{3} \times S^{7}$ vacuum of Sugimoto's $U S p(32)$ open string theory is unstable. This explains, at a heuristic level, the apparent absence of a heterotic string dual.
\end{abstract}

August 2001 


\section{Introduction}

The Breitenlohner-Freedman (BF) bound [1] (see also [2, 3, 4]) says that tachyons in anti-de Sitter space (AdS) cause an instability only if their mass-squared falls below a certain negative threshold, of order the reciprocal of the AdS radius squared. In the AdS/CFT correspondence [5, 6, 7] (for a review see [8]), AdS vacua with some field(s) violating the BF bound need not have a well-defined field theory dual. Indeed, if one attempts to compute the two-point function of such a field, the result is highly cutoff-dependent. This is like having a lattice theory without a well-defined continuum limit. By extension, solutions to string theory or supergravity which are asymptotic to AdS vacua violating the bound may also be expected to have some pathology on the field theory side.

Supersymmetry guarantees that the BF bound will be satisfied, but it is well-known that the converse is not true. For instance, there is an infinite family of compactifications of M-theory, $A d S_{4} \times M^{p q r}$, which are stable but non-supersymmetric [9]. $M^{p q r}$ is a homogeneous Einstein 7-manifold describable as a coset space $(S U(3) \times S U(2) \times$ $U(1)) /(S U(2) \times U(1) \times U(1))$. The integers $p, q$, and $r$ describe the embedding of $S U(2) \times U(1) \times U(1)$ in $S U(3) \times S U(2) \times U(1)$. In most cases the symmetry group of these spaces is $S U(3) \times S U(2) \times U(1) .{ }^{1}$ For a certain range of $p, q$, and $r$, there is a $\mathrm{BF}$-violating tachyon, and for the complementary range there is not.

A question many times raised, but never (as far as we know) seriously investigated, is whether a similar phenomenon occurs for $A d S_{5} \times T^{p q}$ compactifications. It is wellunderstood that the $A d S_{5} \times T^{11}$ case is stable, since it is the supersymmetric nearhorizon limit of D3-branes on a conifold [10]. Also, $A d S_{5} \times T^{k k}$ must be stable (at least classically) since it is a smooth, supersymmetry-breaking $\mathbf{Z}_{k}$ quotient of $T^{11}$, as we shall review further below. On the other hand, $T^{01}$ is a direct product space, $S^{2} \times S^{3}$, and such product geometries are always unstable toward inflating one factor while deflating the other, provided the total number of compact dimensions is less than nine $[11,12]$. The question then is whether $T^{p q}$ is stable for some range of $p / q$ close to 1 , as occurs in the $M^{p q r}$ case. In fact it is not: we shall demonstrate that all $T^{p q}$ for $p \neq q$ are unstable by constructing the unstable mode explicitly. To a large extent this dashes the hope that renormalization group flows from the simplest $\mathbf{Z}_{2}$ orbifold of four-dimensional $\mathcal{N}=4$ gauge theory might include infinitely many infrared fixed points. $^{2}$ (There is still the possibility that stable solutions exist with topology $S^{2} \times S^{3}$ and with three-form field strengths and non-trivial dilaton; however these seem much more difficult to find).

\footnotetext{
${ }^{1}$ The two exceptions are $M^{101}=S^{5} \times S^{2}$ and $M^{011}=C P^{2} \times S^{3}$.

${ }^{2}$ The $M^{p q r}$ manifolds are topologically distinct from one another for different values of $\frac{p}{q}$, so there is no question of whether one could flow from one to another.
} 
A second question which we address is the stability of the $A d S_{3} \times S^{7}$ vacuum of Sugimoto's $U S p(32)$ open string theory [13]. The reason to be interested in this vacuum is that it is the near-horizon limit of many coincident D1-branes in this theory. For the usual $S O(32)$ open string, the D1-brane turns out to be a non-perturbative construction of the $S O(32)$ heterotic string $[14,15]$. There is no perturbative $U S p(32)$ heterotic string in ten dimensions: the $U S p(32)$ current algebra is too big to admit unitary representations with $c \leq 16$. Correspondingly, it is perhaps satisfying that we find fields which violate the BF bound in the $A d S_{3} \times S^{7}$ vacuum of Sugimoto's theory. One may perhaps draw the general conclusion that string theories which are non-supersymmetric in their perturbative construction can suffer non-perturbative instabilities which prevent them from participating in weak-strong coupling dualities.

The organization of the paper is as follows. After some introductory remarks on Freund-Rubin compactifications in section 2, we present the stability analysis for the $T^{p q}$ backgrounds in section 3 . In section 4 we discuss candidate field theory operators of dimension 2 which are dual to the modes of the supergravity analysis which just saturate the stability bound for $T^{11}$. After this, we proceed to the $A d S_{3} \times S^{7}$ background of $U S p(32)$ open string theory in section 5 .

\section{Freund-Rubin Backgrounds}

In this section, for completeness and to fix notation, we review the Freund-Rubin background. We start out by considering classical $D=p+q$ dimensional gravity theory coupled to a $q$-form field strength. The action is given by:

$$
S=\int d^{p} x d^{q} y \sqrt{-g}\left(R-\frac{1}{2 q !} F_{q}^{2}\right),
$$

which leads to the equations of motion:

$$
\begin{gathered}
R_{M N}=\frac{1}{2(q-1) !} F_{M P_{2} \cdots P_{q}} F_{N}{ }^{P_{2} \cdots P_{q}}-\frac{(q-1)}{2(D-2) q !} g_{M N} F_{q}^{2} \\
d * F_{q}=0 .
\end{gathered}
$$

This theory supports a Freund-Rubin solution with the product metric:

$$
d s^{2}=d s_{A d S_{p}}^{2}+d s_{M_{q}}^{2}
$$

describing a product of $p$-dimensional anti-de Sitter space with an Einstein manifold:

$$
R_{\mu \nu}=-\frac{(p-1)}{L^{2}} g_{\mu \nu}
$$




$$
R_{\alpha \beta}=\frac{(q-1)}{R^{2}} g_{\alpha \beta}
$$

and a background field strength on the compact space:

$$
F_{q}=e \operatorname{vol}\left(M_{q}\right) .
$$

We use $M, N, \ldots$ for indices on the full $D$-dimensional spacetime, while $\mu, \nu, \ldots$ are indices on $A d S$ and $\alpha, \beta, \ldots$ are indices on $M_{q}$. The equations of motion (2), (3) relate the length scales $L$ and $R$ and the constant $e$ :

$$
\begin{gathered}
e^{2}=\frac{2(D-2)(q-1)}{(p-1) R^{2}}, \\
L=\frac{p-1}{q-1} R .
\end{gathered}
$$

\section{Stability Analysis for Compactifications on $T^{p q}$}

Let us now examine the issue of stability of Type IIB supergravity compactified on the manifold commonly known as $T^{p q}$. This is a five-dimensional Einstein manifold which is a coset of $S U(2) \times S U(2)$ by a $U(1)$ whose generator can be written as $p \Sigma_{3}+q \tilde{\Sigma}_{3}$, where $\Sigma_{3}$ and $\tilde{\Sigma}_{3}$ are generators of the two $S U(2)$ 's. The integers $p$ and $q$ describe the winding numbers of the $U(1)$ fiber over the two spheres. The most general metric on $T^{p q}$ consistent with $S U(2) \times S U(2) \times U(1)$ isometry is:

$d s^{2}=a^{2}\left(d y_{1}^{2}+\sin ^{2} y_{1} d y_{2}^{2}\right)+b^{2}\left(d y_{3}^{2}+\sin ^{2} y_{3} d y_{4}^{2}\right)+c^{2}\left(d y_{5}-p \cos y_{1} d y_{2}-q \cos y_{3} d y_{4}\right)^{2}$,

where $a, b$, and $c$ are constants, $y_{1}$ and $y_{3}$ range from 0 to $\pi, y_{2}$ and $y_{4}$ range from 0 to $2 \pi$, and $y_{5}$ ranges from 0 to $4 \pi$. Conditions on $a, b$, and $c$ for the metric (10) to be Einstein were discussed in [16], and we will recap some of the relevant points as they will apply to our subsequent analysis. We will assume throughout that $p$ and $q$ are relatively prime, and then in the last paragraph of this section address the case where they are not.

Let us choose the following basis of 1-forms:

$$
\begin{aligned}
& E^{1}=a d y_{1}, \quad E^{2}=a \sin y_{1} d y_{2}, \quad E^{3}=b d y_{3}, \quad E^{4}=b \sin y_{3} d y_{4}, \\
& E^{5}=c\left(d y_{5}-p \cos y_{1} d y_{2}-q \cos y_{3} d y_{4}\right) .
\end{aligned}
$$

The spin-coefficients in this basis are:

$$
\begin{array}{ll}
\omega_{12}=-\frac{1}{a} \cot y_{1} E^{2}-\frac{p c}{2 a^{2}} E^{5}, & \omega_{13}=\omega_{14}=0, \quad \omega_{15}=-\frac{p c}{2 a^{2}} E^{2},
\end{array}
$$


The curvature components are calculated using the relation:

$$
R_{\nu}^{\mu}=d \omega^{\mu}{ }_{\nu}+\omega^{\mu}{ }_{\alpha} \wedge \omega^{\alpha}{ }_{\nu}
$$

Only a few of them need to be actually computed. The remaining ones can be found using the symmetry of the metric and the symmetric and anti-symmetric properties of the Riemann tensor. However, for completeness we list all of the components of the curvature 2-form:

$$
\begin{aligned}
& R_{2}^{1}=\left(\frac{1}{a^{2}}-\frac{3 p^{2} c^{2}}{4 a^{4}}\right) E^{1} E^{2}-\frac{p q c^{2}}{2 a^{2} b^{2}} E^{3} E^{4}, \quad R_{3}^{1}=-\frac{p q c^{2}}{4 a^{2} b^{2}} E^{2} E^{4}, \quad R^{1}{ }_{4}=\frac{p q c^{2}}{4 a^{2} b^{2}} E^{2} E^{3} \\
& R_{4}^{3}=\left(\frac{1}{b^{2}}-\frac{3 q^{2} c^{2}}{4 b^{4}}\right) E^{3} E^{4}-\frac{p q c^{2}}{2 a^{2} b^{2}} E^{1} E^{2}, \quad R^{2}{ }_{4}=-\frac{p q c^{2}}{4 a^{2} b^{2}} E^{1} E^{3}, \quad R^{2}{ }_{3}=\frac{p q c^{2}}{4 a^{2} b^{2}} E^{1} E^{4} \\
& R_{5}^{1}=\frac{p^{2} c^{2}}{4 a^{4}} E^{1} E^{5}, \quad R^{2}{ }_{5}=\frac{p^{2} c^{2}}{4 a^{4}} E^{2} E^{5}, \quad R_{5}^{3}=\frac{q^{2} c^{2}}{4 b^{4}} E^{3} E^{5}, \quad R^{4}{ }_{5}=\frac{q^{2} c^{2}}{4 b^{4}} E^{4} E^{5} .
\end{aligned}
$$

Finally, we have to demand that the metric above is Einstein. In the orthonormal basis that we chose above, this condition is simply that $R^{i}{ }_{j}=\Lambda \delta^{i}{ }_{j}$, where $\Lambda$ is the constant of proportionality between the Ricci tensor and the metric. This yields three equations relating the constants $\Lambda, a, b$ and $c$ :

$$
\Lambda=\frac{2 a^{2}-p^{2} c^{2}}{2 a^{4}}=\frac{2 b^{2}-q^{2} c^{2}}{2 b^{4}}=\frac{\left(a^{4} q^{2}+b^{4} p^{2}\right) c^{2}}{2 a^{4} b^{4}} .
$$

For convenience, let us work in units where the radius of one of the spheres is set equal to unity, i.e. $a \equiv 1$. In these units the other constants $b$ and $c$ are:

$$
b^{2}=\frac{1}{3 \Lambda-1} \quad c^{2}=\frac{2(1-\Lambda)}{p^{2}} .
$$

It is also helpful to express the ratio $\frac{q}{p}$ in terms of $\Lambda$ :

$$
\left(\frac{q}{p}\right)^{2}=\frac{2 \Lambda-1}{(1-\Lambda)(3 \Lambda-1)^{2}} .
$$

Looking at the last expression we see that $\Lambda$ varies between $\frac{1}{2}$ and 1 . So, given any manifold $T^{p q}$ we first evaluate the ratio $\frac{q}{p}$ and then using (17) compute $\Lambda$. For instance, the space $T^{11}$ has $\Lambda=\frac{2}{3}$. All questions about stability can be answered in terms of values of $\Lambda$.

In [12] it was shown that for an arbitrary Einstein manifold, the masses of the scalar modes resulting from a mixing of 3 scalars: the trace of the metric on $A d S_{5}$, the trace on $M_{5}$ and another scalar which arises from the fluctuations of the five form field 
strength never violate the stability bound - they can at most saturate it. The masses of the coupled scalar modes are:

$$
m^{2} L^{2}=\lambda+16 \pm 8 \sqrt{\lambda+4}
$$

where $\square_{y} Y \equiv-\lambda Y / R^{2}$ and $Y$ is a scalar harmonic on $T^{p q}$. By $\square_{y}$ we will always mean $\nabla^{\alpha} \nabla_{\alpha}$. Minimizing with respect to $\lambda$ we find that the least massive mode corresponds to $\lambda=12$. Moreover, this mode just saturates the stability bound $m^{2} L^{2} \geq-4$. The isometry group of $T^{p q}$ is $S U(2) \times S U(2) \times U_{R}(1)$ so the eigenvalues of the scalar Laplacian on $T^{p q}$ are expressed in terms of the eigenvalues $j_{1}, j_{2}, r$ corresponding to the two $S U(2)$ 's and the $U(1)_{R}[17,18,19]$ :

$$
\frac{\lambda}{R^{2}}=\frac{r^{2}}{c^{2}}+\frac{1}{a^{2}}\left[j_{1}\left(j_{1}+1\right)-(p r)^{2}\right]+\frac{1}{b^{2}}\left[j_{2}\left(j_{2}+1\right)-(q r)^{2}\right],
$$

with $j_{1} \geq p r$ and $j_{2} \geq q r$. Let us examine this for the special case of $T^{11}$. Here we have from (17) and (16) $\Lambda=\frac{2}{3}, a=b=1$, and $c^{2}=\frac{2}{3}$. Since $\Lambda=\frac{4}{R^{2}}$ from (6) and from (9) $R=L$, the expression for the eigenvalue of the scalar harmonic on $T^{11}$ simplifies to:

$$
\lambda=6\left[j_{1}\left(j_{1}+1\right)+j_{2}\left(j_{2}+1\right)-\frac{r^{2}}{2}\right] .
$$

The value of $\lambda=12$ is thus satisfied for $\left(j_{1}, j_{2}, r\right)=(1,0,0)$ and $(0,1,0)$.

None of these coupled scalar modes can violate the stability bound for any $T^{p q}$. But, it was shown in [12] that the only mode which could potentially violate the stability bound is the traceless graviton mode. Without proof again, we write down the equation of motion for this fluctuation (for a derivation, the reader is referred to [12]):

$$
\left[\left(\square_{x}+\square_{y}\right) \delta_{c}^{a} \delta_{d}^{b}-2 R_{c}^{a b} d\right]\left(\phi(x) Y_{a b}(y)\right)=0,
$$

where as usual, $\square_{y}=\nabla^{\alpha} \nabla_{\alpha}$ and $\square_{x}=\nabla^{\mu} \nabla_{\mu}$. This equation may be rewritten in terms of the Ricci tensor and the Lichnerowicz operator $\Delta_{L}$. The action of the latter on symmetric tensors $Y_{a b}$ is defined as:

$$
\Delta_{L} Y_{a b} \equiv \square_{y} Y_{a b}-2 R_{a}{ }^{c d}{ }_{b} Y_{c d}-2 R_{(a}^{c} Y_{b) c} .
$$

Here and below, $(\ldots)$ indicates symmetrization: $(a b)=(a b+b a) / 2$. Using the definition (22), the fluctuation equation for the symmetric traceless graviton modes (21) is simply:

$$
\left[\square_{x}+\Delta_{L}+\frac{2(q-1)}{R^{2}}\right]\left(\phi Y_{a b}\right)=0 .
$$

Since we're dealing with $A d S_{5} \times M_{5}, p=q=5$ and from (9) we have $R=L$. The Breitenlohner-Freedman bound is $m^{2} L^{2} \geq-4$. Assembling all these facts together we 
can translate the BF bound from a bound on the mass to one on the eigenvalue of the Lichnerowicz operator acting on symmetric tensors $Y_{a b}$ :

$$
\Delta_{L} Y_{a b}=\lambda Y_{a b}
$$

For stability we must have:

$$
\lambda L^{2} \leq-4
$$

It is somewhat painful to diagonalize the Lichnerowicz operator directly. Fortunately, a trick employed in [9] works here as well. ${ }^{3}$ The key is to use the identity:

$$
\int d V Y^{a b} \Delta_{L} Y_{a b}=\int d V\left[-4 Y^{a b} R_{a c d b} Y^{c d}-4 \Lambda Y^{a b} Y_{a b}-3 \nabla^{(a} Y^{b c)} \nabla_{(a} Y_{b c)}\right]
$$

which can easily be demonstrated by writing out explicitly $Y^{a b} \Delta_{L} Y_{a b}$ and $\nabla^{(a} Y^{b c)} \nabla_{(a} Y_{b c)}$, simplifying, and integrating by parts. Let us diagonalize the Riemann tensor by solving the eigenvalue equation:

$$
R_{a}^{b c}{ }_{d} Y_{b c}=\kappa Y_{a d} .
$$

This would involve diagonalizing a $15 \times 15$ matrix. One of the eigenvectors would be pure trace, so that we'll be left with 14 traceless eigenvectors. Using (25) and (26) our stability bound now reads: The geometry would be stable if every $\kappa$ satisfies:

$$
\kappa \geq \frac{1}{L^{2}}-\Lambda
$$

From (6) we have $\Lambda=\frac{4}{R^{2}}$ and since $R=L$, the above bound can be expressed solely in terms of $\Lambda$ as:

$$
\kappa_{\min } \geq-\frac{3}{4} \Lambda
$$

where $\kappa_{\min }$ is the least of the 14 eigenvalues. Thus we have reduced the problem of solving the complicated equation (21) into a simple one of diagonalizing the Riemann tensor. On account of the simple metric and the symmetries involved, there is very little mixing of the modes and the problem is sufficiently simple to be solved by hand. The eigenvectors and eigenvalues are shown in table 1 . In this table, $\alpha_{ \pm}, \beta_{ \pm}$and $\gamma_{ \pm}$ are constants given by

$$
\begin{aligned}
& \alpha_{ \pm}=4(1-2 \Lambda)\left(7 \Lambda-4 \mp \sqrt{49 \Lambda^{2}-60 \Lambda+20}\right) \\
& \beta_{ \pm}=2(1-2 \Lambda)\left(-7 \Lambda+2 \pm \sqrt{49 \Lambda^{2}-60 \Lambda+20}\right) \\
& \gamma_{ \pm}=\left(2-\Lambda \pm \sqrt{49 \Lambda^{2}-60 \Lambda+20}\right)\left(-7 \Lambda+2 \pm \sqrt{49 \Lambda^{2}-60 \Lambda+20}\right)
\end{aligned}
$$

and we have also defined

$$
X_{a b}^{c d}=\delta_{(a}^{c} \delta_{b)}^{d} .
$$

\footnotetext{
${ }^{3}$ We thank C. Pope for a communication which brought this paper to our attention.
} 


\begin{tabular}{|c|c|c|}
\hline Eigenvectors & Eigenvalues & Eigenvalues in units $a=1$ \\
\hline$X_{a b}^{12}, X_{a b}^{11}-X_{a b}^{22}$ & $\frac{1}{a^{2}}-\frac{3 p^{2} c^{2}}{4 a^{4}}$ & $\frac{1}{2}(3 \Lambda-1)$ \\
$X_{a b}^{34}, X_{a b}^{33}-X_{a b}^{44}$ & $\frac{1}{b^{2}}-\frac{3 q^{2} c^{2}}{4 b^{4}}$ & $\frac{1}{2}$ \\
$X_{a b}^{13}+X_{a b}^{24}, X_{a b}^{14}+X_{a b}^{23}$ & $\frac{3 p q c^{2}}{4 a^{2} b^{2}}$ & $\frac{3}{2} \sqrt{(1-\Lambda)(2 \Lambda-1)}$ \\
$X_{a b}^{13}-X_{a b}^{24}, X_{a b}^{14}-X_{a b}^{23}$ & $-\frac{3 p q c^{2}}{4 a^{2} b^{2}}$ & $-\frac{3}{2} \sqrt{(1-\Lambda)(2 \Lambda-1)}$ \\
$X_{a b}^{15}, X_{a b}^{25}$ & $\frac{p^{2} c^{2}}{4 a^{4}}$ & $\frac{1}{2}(1-\Lambda)$ \\
$X_{a b}^{35}, X_{a b}^{45}$ & $\frac{q^{2} c^{2}}{4 b^{4}}$ & $\left(\Lambda-\frac{1}{2}\right)$ \\
$\alpha_{+}\left(X_{a b}^{11}+X_{a b}^{22}\right)+\beta_{+}\left(X_{a b}^{33}+X_{a b}^{44}\right)+\gamma_{+} X_{a b}^{55}$ & & $\frac{1}{4}\left(-\Lambda+\sqrt{49 \Lambda^{2}-60 \Lambda+20}\right)$ \\
$\alpha_{-}\left(X_{a b}^{11}+X_{a b}^{22}\right)+\beta_{-}\left(X_{a b}^{33}+X_{a b}^{44}\right)+\gamma_{-} X_{a b}^{55}$ & & $\frac{1}{4}\left(-\Lambda-\sqrt{49 \Lambda^{2}-60 \Lambda+20}\right)$ \\
\hline
\end{tabular}

Table 1: Eigenvectors and eigenvalues of the Riemann tensor, as defined in (27).

We have not written down the eigenvalues for the last two modes in the table in terms of $a, b, c, p$ and $q$ because the expressions are quite lengthy. Remembering that $\frac{1}{2} \leq \Lambda \leq 1$ we find that there is only one mode (the last one in the table) which can violate the bound (29). For this potentially dangerous mode we find that only $\Lambda=\frac{2}{3}$ (which corresponds to the manifold $T^{11}$ ) saturates the bound, while all other values of $\Lambda$ lead to masses which violate the bound. This tells us that the only stable compactification on $T^{p q}$ manifolds with $p$ and $q$ relatively prime turns out also to be the only one which preserves supersymmetry. (Note that the modes $X_{a b}^{13}-X_{a b}^{24}$ and $X_{a b}^{14}-X_{a b}^{23}$ saturate the bound for $T^{11}$ while for all other $T^{p q}$ have eigenvalues which are above the bound. This might lead us to suspect that for $T^{11}$ these modes have masses $m^{2} L^{2}=-4$. But on careful examination we find that they do not satisfy the Killing tensor equation $\nabla_{(a} Y_{b c)}=0$. Therefore, according to (26) they have masses $\left.m^{2} L^{2}>-4\right)$.

A word now about what the unstable mode (or in the case of $T^{11}$ the marginally stable mode) looks like. For $T^{11}$, we can use (30) to evaluate the constants $\alpha_{-}=-\frac{8}{3}$, $\beta_{-}=\frac{8}{3}$, and $\gamma_{-}=0$ so that the eigenvector which just saturates the bound is simply $\operatorname{diag}(-1,-1,1,1,0)$. Geometrically this is a fluctuation in which one $S^{2}$ expands while the other shrinks with the length of the $U(1)$ fiber unchanged. For generic $T^{p q}$ however, such a simple picture is not obtained. As an example, let us consider the unstable mode of $T^{12}$. For this manifold, using (17) we find $\Lambda \approx 0.9331$, and using (30) we have 
$\alpha_{-} \approx-17.73, \beta_{-} \approx 12.33$, and $\gamma_{-} \approx 10.80$ so this fluctuation makes one $S^{2}$ shrink and the other expand accompanied by an elongation of the fiber.

To summarize, for $T^{11}$ we have found a total of seven modes which saturate the stability bound. Six of them come from the coupled scalar modes, and the remaining one is a traceless graviton mode. All of these modes have masses $m^{2} L^{2}=-4$. Using the relation between the mass and the dimension of the corresponding operators in the dual field theory, $\Delta=\frac{1}{2}\left[(p-1) \pm \sqrt{(p-1)^{2}+4 m^{2} L^{2}}\right]$ (here $p$ is the dimension of $A d S$ ), we find that the operators have scaling dimension 2. In the next section we shall examine in some detail the issue of identifying these operators in the dual field theory according to the AdS/CFT correspondence.

In the above analysis we have only shown that if the inequality (29) is satisfied, then stability is guaranteed. Let us now prove that if the inequality is violated, then we necessarily have instability in the traceless graviton sector. Looking at (26) we find that we have to demonstrate that the putative unstable mode is also a Killing tensor obeying $\nabla_{(a} Y_{b c)}=0$. To prove this, we make the following observations. First, if we restrict all three indices $a, b$, and $c$ to lie in the four manifold which are the two $S^{2}$ 's of $T^{p q}$ (i.e. these indices are allowed to run from 1 to 4 ), then a constant, diagonal tensor of the form:

$$
Y_{a b}=\operatorname{diag}(\alpha, \alpha, \beta, \beta, \gamma)
$$

with $2 \alpha+2 \beta+\gamma=0$ is covariantly constant. Second, we notice that the spin-coefficients of the metric written down in (12) can be split up in the following way:

$$
\omega_{a b}^{5}=\omega_{a b}^{4}+c_{a b} E^{5} \quad \omega_{a 5}^{5}=c_{a b} E^{b},
$$

where $\omega^{5}$ refers to the full spin connection, $\omega^{4}$ refers to the part on the two $S^{2}$ 's, and $c_{a b}$ is antisymmetric in $a$ and $b$. Using this fact and the form of the constant diagonal tensor, one can show that indeed this eigentensor satisfies the Killing condition. So we have demonstrated that the only stable $A d S_{5} \times T^{p q}$ compactification with $p$ and $q$ relatively prime is on $T^{11}$.

To extend the discussion to the case of $p$ and $q$ not relatively prime, a topological point should be made first: if $\operatorname{gcd}(p, q)=k \neq 1$, then $T^{p q}$ is topologically $S^{2} \times S^{3} / \mathbf{Z}_{\mathbf{k}}$, where the $\mathbf{Z}_{k}$ acts freely on the Hopf fiber of $S^{3}$. The $k=1$ case of this statement follows based on arguments given in [20]; the $k>1$ statement follows as a corollary when one notes that modding out by the $U(1)$ generated by $p \Sigma_{3}+q \tilde{\Sigma}_{3}$ can be accomplished by first modding out by the $U(1)$ generated by $\left(p \Sigma_{3}+q \tilde{\Sigma}_{3}\right) / k$ and then dividing by $\mathbf{Z}_{k}$. Moreover, $T^{p q}$ is metrically a quotient of $T^{p / k, q / k}$ by $\mathbf{Z}_{k}$ acting on the $U(1)$ fiber. Thus one can flow without encountering a topological obstruction from any $T^{p q}$ to any other precisely when $\operatorname{gcd}(p, q)$ remains unchanged. In each class of manifolds $T^{p q}$ with $\operatorname{gcd}(p, q)=k$ fixed, there is precisely one which is classically stable, namely $T^{k k}$. Only for $k=1$ is any supersymmetry preserved. The perturbation analysis on $T^{p q}$ could 
be carried out by considering $\mathbf{Z}_{k}$ invariant functions on $T^{p / k, q / k}$. The orbifolding by $k$ also has a well-defined meaning on the gauge theory side, resulting for $T^{k k}$ in a theory with gauge group $S U(N)^{2 k}$ and some complicated matter. Details of counting and the operator map could be pursued for the case of general $k$, but in the next section we will do so only for $k=1$.

\section{The Operator Map for $T^{11}$}

In the previous section we saw that the Freund-Rubin compactification of Type IIB on $A d S_{5} \times T^{11}$ have three modes which have masses which just saturate the stability bound. Recall that two of these modes came from the coupled scalars and the remaining one is a traceless graviton mode. According to the AdS/CFT correspondence, these modes should correspond to operators whose dimension is protected and would therefore be either chiral primaries or conserved currents. So let us try to find the dual operators.

The compactification of Type IIB SUGRA on $T^{11}$ has $S U(2,2 \mid 1)$ symmetry. Let us try to put these fluctuations into $S U(2,2 \mid 1)$ supermultiplets. A unitary highest weight representation of $S U(2,2 \mid 1)$ can be decomposed into a direct sum of unitary highest weight representations of the bosonic subalgebra $S U(2,2) \times U_{R}(1)$ whose maximal compact subalgebra is $U(1) \times S U(2) \times S U(2) \times U_{R}(1)[21,22]$. The first $U(1)$ is the energy and the last one is the $R$-charge. So the highest weight representations of $S U(2,2 \mid 1)$ are labelled by four quantum numbers $D\left(E_{0}, s_{1}, s_{2} ; r\right)$. In addition to these, there are of course, the quantum numbers associated with the symmetry of the isometry group of $T^{11}$ which is $S U(2) \times S U(2) \times U_{R}(1)$. Note that this $U_{R}(1)$ is the same $U_{R}(1)$ as the one associated to the R-charge. We'll call the additional quantum numbers due to these last two $S U(2)$ 's as $\left(j_{1}, j_{2}\right)$. So our first task is to find out all the six quantum numbers of the fluctuation mode in question $\left(E_{0}, s_{1}, s_{2} ; r, j_{1}, j_{2}\right)$.

From the $5 d$ AdS point of view all of the modes in question, including $Y_{a b}$, are scalars. So, the spin quantum numbers $s_{1}=s_{2}=0$. To compute the AdS energy, we use the relation between mass and energy for a scalar in $5 d$ AdS space:

$$
E_{0}=2 \pm \sqrt{4+m^{2} L^{2}}
$$

We found above that these modes have masses $m^{2} L^{2}=-4$, so $E_{0}=2$. Finally, to obtain the value of $r$, we note that for a representation of $S U(2,2 \mid 1)$ to be unitary, there are inequalities among the four quantum numbers $E_{0}, s_{1}, s_{2}$, and $r$. The relevant one for our purposes here is $E_{0} \geq 2 s_{2}+\frac{3}{2} r+2$ which fixes $r=0$. We observe now that this set of quantum numbers $(2,0,0,0)$ satisfies 3 multiplet shortening conditions [21] (which is what is expected for a field saturating the unitarity bound):

$$
E_{0}-2 s_{1}+\frac{3}{2} r-2=0 \quad E_{0}-2 s_{2}+\frac{3}{2} r-2=0 \quad s_{2}=0 .
$$


So we get the following multiplet with only 4 fields present:

\begin{tabular}{|c|c|c|c|}
\hline$E_{0} / R$ & $r=-1$ & $r=0$ & $r=1$ \\
\hline 2 & & $(0,0)$ & \\
$\frac{3}{2}$ & $\left(\frac{1}{2}, 0\right)$ & & $\left(0, \frac{1}{2}\right)$ \\
3 & & $\left(\frac{1}{2}, \frac{1}{2}\right)$ & \\
\hline
\end{tabular}

where the quantities in the table refer to the quantum numbers $\left(s_{1}, s_{2}\right)$. For completeness, we note that the masses of these fields can be calculated using the relations:

$$
\begin{array}{rlrl}
\left(\frac{1}{2}, 0\right)\left(0, \frac{1}{2}\right) & m & =\left|E_{0}-2\right|=\frac{1}{2}, \\
\left(\frac{1}{2}, \frac{1}{2}\right) & m^{2}=\left(E_{0}-1\right)\left(E_{0}-3\right)=0 .
\end{array}
$$

Let us now turn to the field theory realization of this. The conformal field theory dual to the supergravity theory has two doublets of chiral superfields $A_{i}, B_{j}(i, j=1,2)$ transforming in the $(N, \bar{N})$ and $(\bar{N}, N)$ representations of $S U(N) \times S U(N)$. These fields both have $R$-charge 1 . The global symmetry group $S U(2) \times S U(2)$ quantum numbers for these fields are $\left(\frac{1}{2}, 0\right)$ and $\left(0, \frac{1}{2}\right)$, respectively. According to the AdS/CFT correspondence, each supergravity field with quantum numbers $\left(E_{0}, s_{1}, s_{2} ; r\right)$ is mapped to a conformal field with scaling dimension $\Delta=E_{0}$, Lorentz quantum numbers of an $S L(2, C)$ representation $\left(s_{1}, s_{2}\right)$, and an $R$-symmetry charge $r$. Since we had multiplet shortening, we know that the dimension of the corresponding superfield would be protected, and furthermore we also determined its dimension to be 2. There are natural field theory candidates with the desired properties to be dual to the scalars we have found. ${ }^{4}$ Namely, consider the real superfields: ${ }^{5}$

$$
\begin{aligned}
J_{A} & =\operatorname{Tr} A_{(i} e^{V_{2}} A_{j)}^{*} e^{V_{1}} \quad J_{B}=\operatorname{Tr} B_{(i} e^{V_{1}} B_{j)}^{*} e^{V_{2}} \\
J_{\text {baryon }} & =\operatorname{Tr} A_{[1} e^{V_{2}} A_{2]}^{*} e^{V_{1}}-\operatorname{Tr} B_{[1} e^{V_{1}} B_{2]}^{*} e^{V_{2}} .
\end{aligned}
$$

The vector component of each of these is a conserved current, by Noether's theorem: $J_{A}$ is associated with the global $S U(2)$ rotating $A_{1}$ and $A_{2} ; J_{B}$ is associated with the other global $S U(2)$; and $J_{\text {baryon }}$ is associated with the unbroken $U(1)_{\text {baryon. The scalar }}$ component of each of these superfields, call them $\mathcal{O}_{A}, \mathcal{O}_{B}$, and $\mathcal{O}_{\text {baryon }}$, have protected

\footnotetext{
${ }^{4}$ We thank M. Strassler for useful communications which helped us identify the field theory operators.

${ }^{5}$ In (37), $V_{1}$ and $V_{2}$ are the real superfields that include the $S U(N) \times S U(N)$ gauge fields. There is a notational subtlety: $A_{i}^{*}$ transforms as a doublet of $S U(2)$, and we have omitted the $\epsilon_{i j}$ which would usually be inserted to make the group action come out right.
} 
dimension 2 and R-charge 0 . The operator $\mathcal{O}_{\text {baryon }}$ was discussed in [23] in the context of resolving the conifold. Of the supergravity modes with masses $m^{2} L^{2}=-4$, we conjecture that the baryon current is the one which is dual to the traceless graviton mode, while $J_{A}$ and $J_{B}$ are dual to the coupled scalar modes. The global $S U(2) \times S U(2)$ charges support this expectation. Moreover, both the traceless graviton fluctuation that we examined in the previous section and its proposed operator dual $J_{\text {baryon }}$ flip sign on interchanging the $S^{2}$ s, i.e. they are both $\mathbf{Z}_{2}$ odd.

\section{Sugimoto's $U S p(32)$ open string theory}

The next example of an unstable Freund-Rubin compactification that we shall consider arises in the $U S p(32)$ open string theory considered in [13]. As we shall discover, the modes which are unstable come from a mixing of the trace of the metric on $A d S_{3}$, the trace on $S^{7}$, and another scalar arising from the fluctuations of the form field. Thus, this instability is of the same type as the one for the Freund-Rubin compactification of massive Type IIA supergravity on $A d S_{4} \times S^{6}$ [12]. The low-energy effective action of the Sugimoto theory in the string frame is [24]:

$$
S=\frac{1}{2 \kappa^{2}} \int d^{10} x \sqrt{G}\left[e^{-2 \phi}\left(R+4(\partial \phi)^{2}\right)-\frac{1}{12} F_{3}^{2}-\alpha e^{-\phi}\right] .
$$

where $\alpha$ for our purposes is just a constant. In our conventions, $F_{3}^{2}=F_{M N P} F^{M N P}$. To bring the action (38) into Einstein frame, we rescale the metric as $g_{M N}=e^{\frac{-\phi}{2}} G_{M N}$. The action then becomes:

$$
S=\frac{1}{2 \kappa^{2}} \int d^{10} x \sqrt{g}\left[R-\frac{1}{2}(\partial \phi)^{2}-\alpha e^{\frac{3}{2} \phi}-\frac{1}{12} e^{\phi} F_{3}^{2}\right] .
$$

The scalar equation of motion which follows from this action is:

$$
\square \phi-\frac{3}{2} \alpha e^{\frac{3}{2} \phi}-\frac{1}{12} e^{\phi} F_{3}^{2}=0 .
$$

For a constant $\phi$ background, this equation can have a solution if we use the FreundRubin ansatz $F_{\mu \nu \rho}=f \epsilon_{\mu \nu \rho}$, i.e. the three-form is along the $A d S$ part. So we find that $A d S_{3} \times S^{7}$ is indeed a solution with $\phi=0$.

For convenience, let us dualize the three-form and use a seven-form instead. The action (which is what we shall be using from now on) is:

$$
S=\frac{1}{2 \kappa^{2}} \int d^{10} x \sqrt{g}\left[R-\frac{1}{2}(\partial \phi)^{2}-\frac{1}{2 \cdot 7 !} e^{-\phi} F_{7}^{2}-\alpha e^{\frac{3}{2} \phi}\right] .
$$

The background geometry has $\phi=0$. The equations of motion are:

$$
R_{M N}=\frac{1}{2 \cdot 6 !} e^{-\phi} F_{M P_{1} \cdots P_{5}} F_{N} P_{1} \cdots P_{5}-\frac{3}{8 \cdot 7 !} e^{-\phi} F_{7}^{2} g_{M N}+\frac{1}{2} \partial_{M} \phi \partial_{N} \phi+\frac{1}{8} \alpha e^{\frac{3}{2} \phi} g_{M N}
$$




$$
\begin{gathered}
d *\left(e^{-\phi} F_{7}\right)=0, \\
\square \phi+\frac{1}{2 \cdot 7 !} e^{-\phi} F_{7}^{2}-\frac{3}{2} \alpha e^{\frac{3}{2} \phi}=0 .
\end{gathered}
$$

We want to express all the parameters in terms of the $A d S$ radius $L$. For the background $F_{7}=c \operatorname{vol}_{S^{7}}$ and $\phi=0$ so (44) gives $c^{2}=\frac{1}{7 !} F_{7}^{2}=3 \alpha$. The Einstein equation yields $\alpha=\frac{2}{L^{2}}=\frac{12}{R^{2}}$. So, the ratio of the radii is $R^{2}=6 L^{2}$. Let us now proceed to get the mass spectrum of the scalars. Tracing over the indices on the sphere in (42) gives:

$$
R_{\alpha}^{\alpha}=\frac{7}{4 L^{2}} e^{\frac{3}{2} \phi}+\frac{7}{8 \cdot 7 !} e^{-\phi} F_{7}^{2}+\frac{1}{2} \partial^{\alpha} \phi \partial_{\alpha} \phi
$$

When expanded to linear order, the two sides of the above equation yield:

$$
-\frac{1}{L^{2}} \pi-\frac{1}{2}\left(\square_{x}+\square_{y}\right) \pi=-\frac{21}{8 L^{2}} \delta \phi+\frac{7}{4} c \square_{y} b-\frac{21}{4 L^{2}} \pi \text {. }
$$

where $\pi$ denotes the trace of the metric fluctuation on $S^{7}$, and the fluctuation of the 7 -form field strength is expressed as $\delta F_{7}=d a_{6}$ with $a_{6}=*_{7} d b$. On simplification this finally gives:

$$
\left(\square_{x}+\square_{y}\right) \pi-\frac{17}{2 L^{2}} \pi+21 \square_{y} B-\frac{21}{4 L^{2}} \delta \phi=0,
$$

where we've introduced the notation $b \equiv c L^{2} B$. For the scalar fluctuations we expand (44) to linear order:

$$
\left(\square_{x}+\square_{y}\right) \delta \phi-\frac{15}{2 L^{2}} \delta \phi-\frac{3}{L^{2}} \pi+6 \square_{y} B=0 .
$$

The form equation (43) expanded to linear order yields after a little algebra:

$$
\left(\square_{x}+\square_{y}\right) B-\frac{6}{7 L^{2}} \pi-\frac{1}{L^{2}} \delta \phi=0 .
$$

Assembling all the three equations, and assuming that $B, \pi$, and $\delta \phi$ are eigenvectors of $\square_{y}$ with eigenvalue $-\lambda / R^{2}$, we obtain the following mass matrix equation:

$$
\square_{x}\left(\begin{array}{c}
B \\
\pi \\
\delta \phi
\end{array}\right)=\left(\begin{array}{ccc}
\frac{\lambda}{R^{2}} & \frac{6}{7 L^{2}} & \frac{1}{L^{2}} \\
\frac{21 \lambda}{R^{2}} & \frac{\lambda}{R^{2}}+\frac{17}{2 L^{2}} & \frac{21}{4 L^{2}} \\
\frac{6 \lambda}{R^{2}} & \frac{3}{L^{2}} & \frac{\lambda}{R^{2}}+\frac{15}{2 L^{2}}
\end{array}\right)\left(\begin{array}{c}
B \\
\pi \\
\delta \phi
\end{array}\right) .
$$

On diagonalizing the matrix, and using the relation $R^{2}=6 L^{2}$ to eliminate $R^{2}$, we obtain the eigenvalues (mass squared) $m^{2} L^{2}=\frac{\lambda+24}{6}, \frac{\lambda+36+12 \sqrt{\lambda+9}}{6}$, and $\frac{\lambda+36-12 \sqrt{\lambda+9}}{6}$. Only states in the last tower can be tachyonic. On $S^{7}$, the spherical harmonics have eigenvalues $\lambda=k(k+6)$. The dangerous tower of states when expressed in terms of $k$ 
become, $m^{2} L^{2}=\frac{k(k-6)}{6}$. Remembering that the BF bound for this system is $m^{2} L^{2} \geq$ -1 , we see that the modes $k=2,3$, and 4 violate the bound. The presence of three unstable modes makes it considerably more difficult to find a stable compactification where $S^{7}$ is replaced by some other seven-manifold $M_{7}$ : there is a fairly wide range of eigenvalues for the laplacian on $M_{7}$ which would lead through (50) to an unstable mode.

\section{Conclusions}

We have investigated two types of anti-de Sitter compactifications and found them to be unstable in the sense of Breitenlohner and Freedman. In the context of AdS/CFT, this instability implies that neither the AdS background nor solutions asymptotic to it at infinity have a unitary field theory dual. To arrive at a solution of supergravity that does have such a dual, it would be necessary to change the asymptotics of the spacetime.

The first class of AdS compactifications we studied is $A d S_{5} \times T^{p q}$. Except for the case $p=q$, we have found these solutions to be unstable, and the unstable mode which we were able to construct is a metric fluctuation under which one $S^{2}$ of $T^{p q}$ expands, the other shrinks, and the $U(1)$ fiber either elongates or shrinks by an amount required to keep the total volume of the manifold constant. Thus it still remains to find a stable, non-supersymmetric anti-de Sitter compactification of type IIB supergravity which is not locally isometric to a supersymmetric one. Infinitely many such compactifications of eleven-dimensional supergravity to $A d S_{4} \times M_{7}$ have long been known, as we remarked in the introduction. This problem of non-supersymmetric $A d S_{5}$ vacua takes on a new interest in light of AdS/CFT, because it corresponds to discovering four-dimensional, non-supersymmetric, strong-coupling fixed points of the renormalization group.

The other compactification which we studied is the $A d S_{3} \times S^{7}$ solution to Sugimoto's USp (32) open string theory. Here the field theory dual, if it existed, would be the CFT describing many coincident dual heterotic strings. A heterotic dual does not seem to exist for this non-supersymmetric open string theory, and so it is sensible that we again find violations of the Breitenlohner-Freedman bound. This may lead us to wonder anew to what extent the duality web of M-theory depends on supersymmetry.

\section{Acknowledgments}

We would like to thank D. Freedman, I. Klebanov, and M. Strassler for useful discussions, and C. Pope for bringing the useful results of [9] to our attention. We also thank D. Freedman and C. Pope for comments on an early draft. This work was supported 
in part by the DOE under grants DE-FG03-92ER40701 and DE-FG02-91ER40671 and through an Outstanding Junior Investigator Award.

\section{References}

[1] P. Breitenlohner and D. Z. Freedman, "Positive energy in Anti-de Sitter backgrounds and gauged extended supergravity," Phys. Lett. B115 (1982) 197.

[2] L. Mezincescu and P. K. Townsend, "Stability at a local maximum in higher dimensional Anti-de Sitter space and applications to supergravity," Ann. Phys. 160 (1985) 406.

[3] P. Breitenlohner and D. Z. Freedman, "Stability in gauged extended supergravity," Ann. Phys. 144 (1982) 249.

[4] P. K. Townsend, "Positive energy and the scalar potential in higher dimensional (super)gravity theories," Phys. Lett. B148 (1984) 55.

[5] J. Maldacena, "The large N limit of superconformal field theories and supergravity," Adv. Theor. Math. Phys. 2 (1998) 231-252, hep-th/9711200.

[6] S. S. Gubser, I. R. Klebanov, and A. M. Polyakov, "Gauge theory correlators from non-critical string theory," Phys. Lett. B428 (1998) 105-114, hep-th/9802109.

[7] E. Witten, "Anti-de Sitter space and holography," Adv. Theor. Math. Phys. 2 (1998) 253-291, hep-th/9802150.

[8] O. Aharony, S. S. Gubser, J. Maldacena, H. Ooguri, and Y. Oz, "Large N field theories, string theory and gravity," Phys. Rept. 323 (2000) 183-386, hep-th/9905111.

[9] D. N. Page and C. N. Pope, "Stability analysis of compactifications of D = 11 supergravity with SU(3) x SU(2) x U(1) symmetry," Phys. Lett. B145 (1984) 337.

[10] I. R. Klebanov and E. Witten, "Superconformal field theory on threebranes at a Calabi-Yau singularity," Nucl. Phys. B536 (1998) 199-218, hep-th/9807080.

[11] M. J. Duff, B. E. Nilsson, and C. N. Pope, "The Criterion For Vacuum Stability In Kaluza-Klein Supergravity," Phys. Lett. B139 (1984) 154. 
[12] O. DeWolfe, D. Z. Freedman, S. S. Gubser, G. T. Horowitz, and I. Mitra, "Stability of $\operatorname{AdS}(p)$ x M(q) compactifications without supersymmetry," hep-th/0105047.

[13] S. Sugimoto, "Anomaly cancellations in type I D9-D9-bar system and the USp(32) string theory," Prog. Theor. Phys. 102 (1999) 685, hep-th/9905159.

[14] J. A. Harvey and A. Strominger, "The heterotic string is a soliton," Nucl. Phys. B449 (1995) 535-552, hep-th/9504047.

[15] J. Polchinski and E. Witten, "Evidence for Heterotic - Type I String Duality," Nucl. Phys. B460 (1996) 525-540, hep-th/9510169.

[16] L. J. Romans, "New compactifications of chiral $N=2, d=10$ supergravity," Phys. Lett. B153 (1985) 392-396.

[17] D. P. Jatkar and S. Randjbar-Daemi, "Type IIB string theory on AdS(5) x T(nn')," Phys. Lett. B460 (1999) 281-287, hep-th/9904187.

[18] S. S. Gubser, "Einstein manifolds and conformal field theories," Phys. Rev. D59 (1999) 025006, hep-th/9807164.

[19] A. Ceresole, G. Dall'Agata, R. D'Auria, and S. Ferrara, "Superconformal field theories from IIB spectroscopy on $\operatorname{AdS}(5)$ x T(11)," Class. Quant. Grav. 17 (2000) 1017-1025, hep-th/9910066.

[20] P. Candelas and X. C. de la Ossa, "COMments On CONIFOLDS," Nucl. Phys. B342 (1990) 246-268.

[21] D. Z. Freedman, S. S. Gubser, K. Pilch, and N. P. Warner, "Renormalization group flows from holography: supersymmetry and a c-theorem," Adv. Theor. Math. Phys. 3 (1999) 363-417, hep-th/9904017.

[22] M. Flato and C. Fronsdal, "Representations Of Conformal Supersymmetry," Lett. Math. Phys. 8 (1984) 159.

[23] I. R. Klebanov and E. Witten, "AdS/CFT correspondence and symmetry breaking," Nucl. Phys. B556 (1999) 89-114, hep-th/9905104.

[24] E. Dudas and J. Mourad, "Consistent gravitino couplings in non-supersymmetric strings," Phys. Lett. B514 (2001) 173-182, hep-th/0012071. 